\title{
HEMANGIOMA VERTEBRAL SINTOMÁTICO TRATADO COM RADIOTERAPIA EXCLUSIVA - RELATO DE CASO E REVISÃO DA LITERATURA*
}

\author{
Douglas Guedes de Castro ${ }^{1}$, Renato Pierre Lima ${ }^{1}$, Maria Aparecida Conte Maia ${ }^{2}$, \\ Paulo Eduardo Ribeiro Santos Novaes ${ }^{3}$, Ricardo César Fogaroli ${ }^{4}$, Robson Ferrigno ${ }^{5}$, \\ Antonio Cássio Assis Pellizzon², João Victor Salvajoli ${ }^{6}$
}
Resumo Neste trabalho descrevemos a apresentação clínica e dos exames de imagem, bem como a utilização da radioterapia exclusiva, em um caso de hemangioma vertebral sintomático. Após três meses de seguimento, houve melhora parcial da dor. Recomendamos dose total de $40 \mathrm{~Gy}$, em frações diárias de $2 \mathrm{~Gy}$. Em seguida é apresentada revisão da literatura.
Unitermos: Hemangioma. Radioterapia. Coluna vertebral.

\begin{abstract}
Treatment of symptomatic vertebral hemangioma by radiotherapy-case report and review of the literature. We report the clinical and imaging findings of a patient with a symptomatic vertebral hemangioma treated with radiotherapy alone. There was partial relief of the pain 3 months after radiotherapy. We recommend a total dose of $40 \mathrm{~Gy}$ at $2 \mathrm{~Gy}$ per fraction. A review of literature is also presented.

Key words: Hemangioma. Radiotherapy. Spine.
\end{abstract}

\section{INTRODUÇÃO}

O hemangioma é uma proliferação vascular benigna que pode ser capilar ou cavernosa. O hemangioma vertebral geralmente é assintomático e descoberto incidentalmente, sendo relatada incidência de $11 \%$ em uma série de necropsias ${ }^{(\mathbf{1})}$. Quando sintomático, a apresentação mais freqüente ocorre sob a forma de dorsalgia e, em alguns casos, pode estar associada a compressão radicular ou medular, causando déficits sensitivo e motor ${ }^{(2)}$.

* Trabalho realizado no Departamento de Radioterapia do Hospital do Câncer A.C. Camargo, São Paulo, SP.

1. Médicos Residentes do Departamento de Radioterapia do Hospital do Câncer A.C. Camargo.

2. Médicos Radioterapeutas do Departamento de Radioterapia do Hospital do Câncer A.C. Camargo, Mestres em Medicina pela FMUSP.

3. Médico Radioterapeuta do Departamento de Radioterapia do Hospital do Câncer A.C. Camargo, Doutor em Medicina pela FMUSP, Professor Titular da Disciplina de Oncologia da Faculdade de Medicina da Unimes.

4. Médico Radioterapeuta do Departamento de Radioterapia do Hospital do Câncer A.C. Camargo.

5. Médico Radioterapeuta do Departamento de Radioterapia do Hospital do Câncer A.C. Camargo, Doutor em Medicina pela FMUSP

6. Médico Radioterapeuta, Diretor do Departamento de Radioterapia do Hospital do Câncer A.C. Camargo, Doutor em Medicina pela Unifesp/EPM, Professor Assistente da Faculdade de Medicina de Marília.

Endereço para correspondência: Dr. Douglas Guedes de Castro. Rua Tamandaré, 997, apto. 1106. São Paulo, SP, 01525-001. E-mail: dougepm@uol.com.br

Recebido para publicação em 17/10/2001. Aceito, após revisão, em 3/12/2001
Na radiografia simples os hemangiomas caracterizam-se por apresentar trabeculados verticais paralelos nos corpos vertebrais. A tomografia computadorizada (TC) revela a presença de trabéculas espessas e áreas radiotransparentes, e a ressonância magnética (RM) apresenta sinais hiperintensos em T1 e T2 ${ }^{(3)}$.

A radioterapia pode ser utilizada no tratamento dos hemangiomas vertebrais de forma exclusiva ou associada à cirurgia. Há poucos relatos na literatura sobre a utilização de radioterapia exclusiva em hemangiomas vertebrais, e a revisão dessas publicações prévias demonstra a variabilidade de esquemas propostos em casos isolados ou pequenas séries ${ }^{(\mathbf{4 - 8})}$.

Neste relato descrevemos a apresentação clínica e dos exames de imagem, e a utilização da radioterapia exclusiva em um caso de hemangioma vertebral, e complementamos com revisão da literatura.

\section{RELATO DO CASO}

Paciente do sexo feminino, 31 anos de idade, com história de dorsalgia de caráter progressivo e sem irradiação há cerca de um ano. Fez uso de antiinflamatório nãohormonal e colete ortopédico para correção postural, porém não houve melhora do quadro, sendo então encaminhada para avaliação de radioterapia. Apresentava exame físico normal à admissão.

A radiografia da coluna vertebral (Figura 1) mostrava estrias verticais formadas por traves ósseas dissociadas nos corpos vertebrais de T12 e L2.

A TC da coluna (Figura 2) evidenciava áreas na medula do corpo vertebral de T12 e L2 com trabeculações esparsas e faixas hipoatenuantes alternadas sem sinais de lesão cortical ou lesão infiltrativa. Mostrava

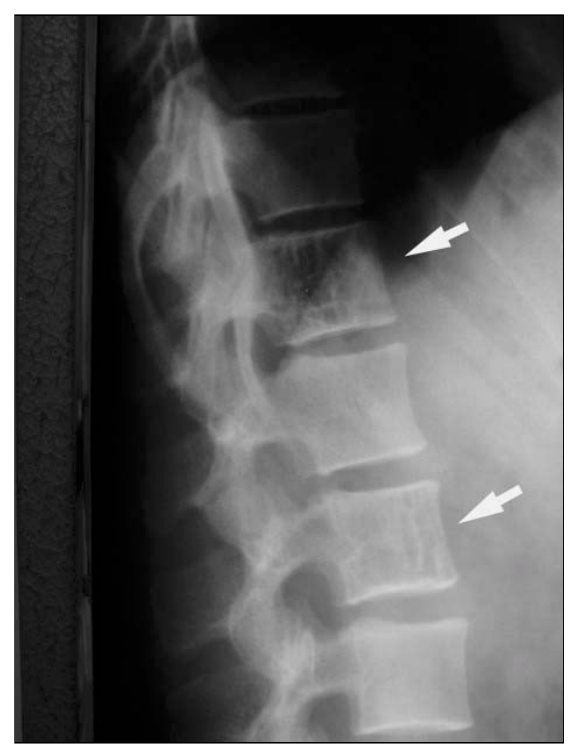

Figura 1. Radiografia da coluna vertebral, em perfil, mostrando estrias verticais típicas. 


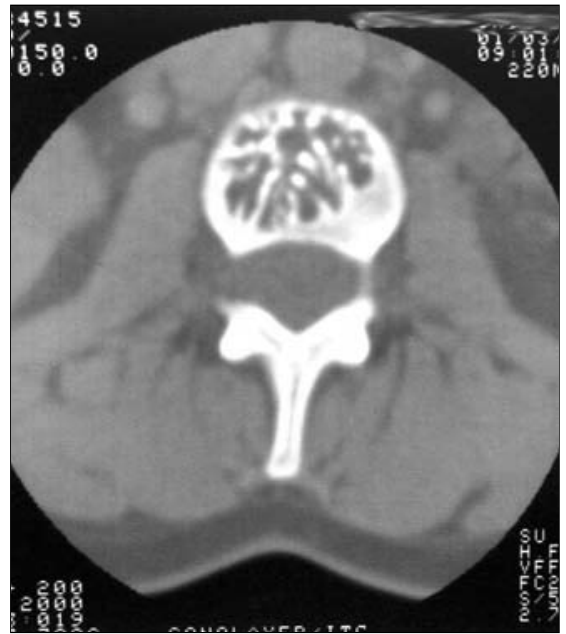

Figura 2. Tomografia computadorizada, corte axial, mostrando faixas hiperatenuantes com trabéculas esparsas na vértebra T12.

características compatíveis com hemangioma vertebral de T12 e L2.

A RM da coluna (Figura 3) mostrava extensa alteração do trabeculado ósseo medular dos corpos vertebrais de T12 e L2, comprometendo inclusive o pedículo esquerdo de T12, caracterizada por alto sinal em T1 e T2, entremeado por focos de ausência de sinal em todas as sequiências. Esses focos eram sugestivos de padrão vascular e apresentaram certo grau de realce após o uso de contraste paramagnético. As alterações eram sugestivas de hemangioma ósseo de T12 e L2.

A paciente foi submetida a radioterapia exclusiva com fótons de $4 \mathrm{MV}$ em três campos posteriores de T11 a L3 (Figura 4). Utilizou-se técnica isocêntrica com um campo posterior direto (peso 1) e dois campos oblíquos de $60^{\circ}$ (peso 0,6 ) com filtro em cunha de $60^{\circ}$. A dose total proposta foi de 40 Gy em 20 frações de 2 Gy e normalizada no isocentro, ou seja, a $7 \mathrm{~cm}$ de profundidade em relação ao dorso. Optou-se pela curva de isodose de 98\% (Figura 5).

\section{DISCUSSÃO}

Dentre os hemangiomas ósseos, a coluna vertebral é o sítio mais freqüentemente acometido, correspondendo a $20 \%$ dos ca$\operatorname{sos}^{(8)}$. A coluna torácica e a lombar são os locais mais acometidos pelos hemangiomas vertebrais ${ }^{(9)}$. O envolvimento multifocal da coluna vertebral é observado em cerca de $30 \%$ dos casos descritos na litera-

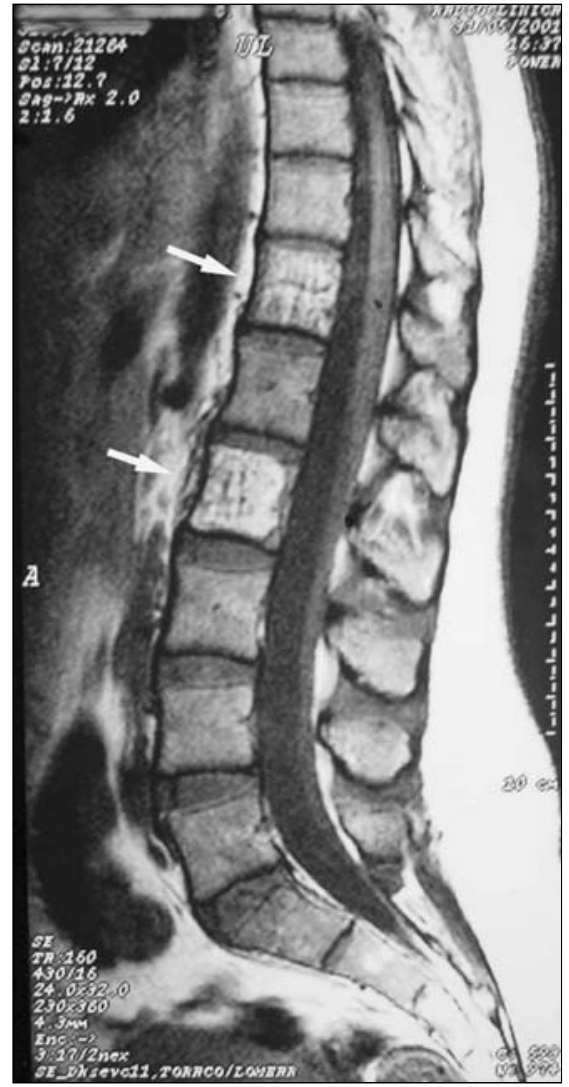

Figura 3. Ressonância magnética, seqüência sagital ponderada em $\mathrm{T} 1$, mostrando sinais hiperintensos em T12 e L2.

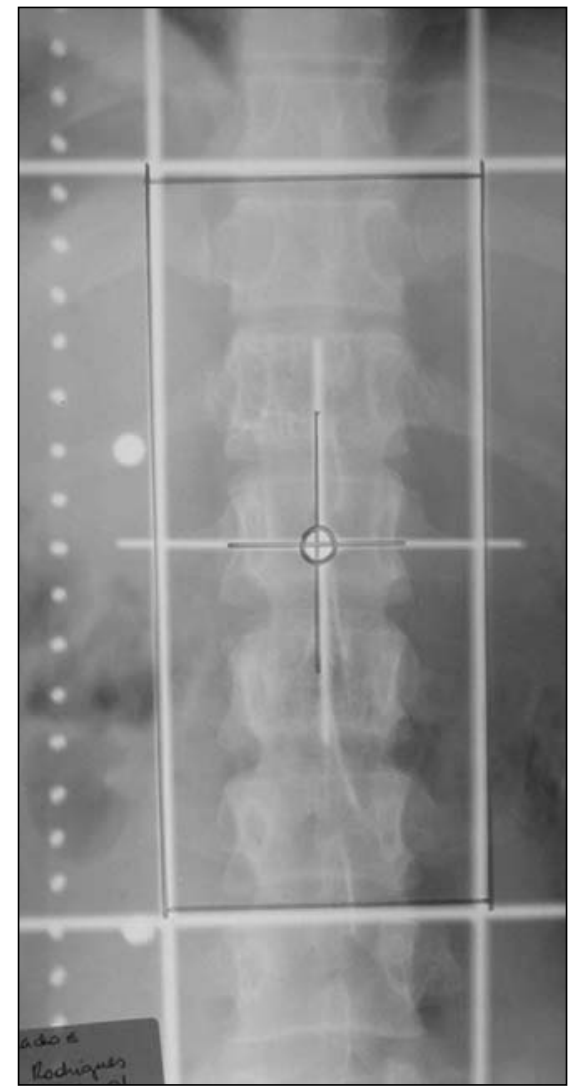

Figura 4. Radiografia de simulação do campo posterior direito envolvendo de T11 a L3.

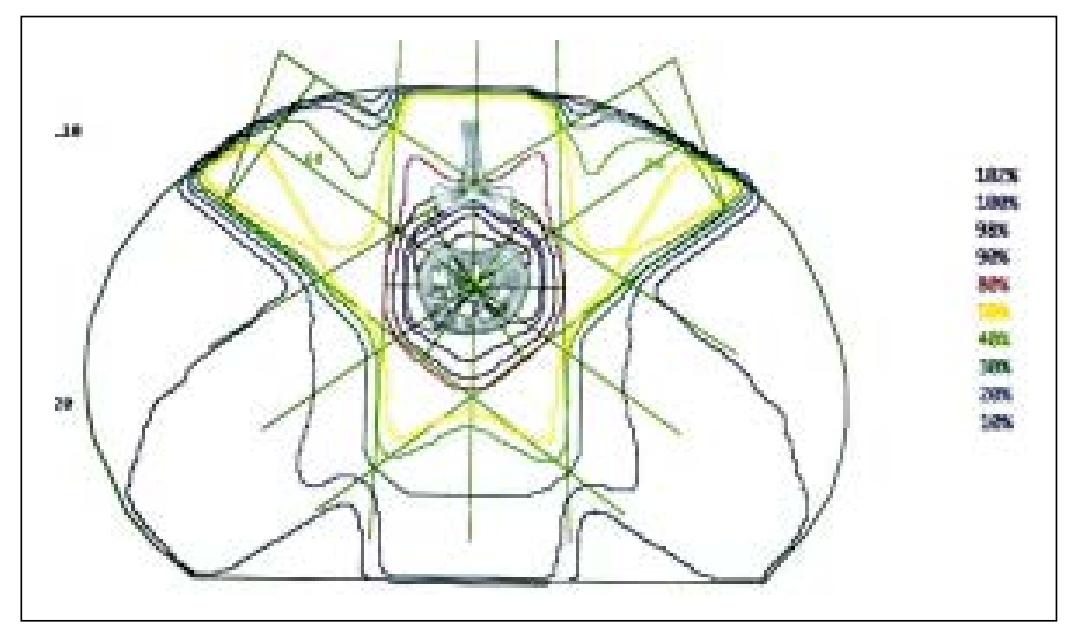

Figura 5. Distribuição das curvas de isodose. Optou-se pela curva de isodose de $98 \%$. tura e, geralmente, há o envolvimento de dois a cinco níveis não-contíguos ${ }^{(\mathbf{1 0})}$. No caso ora relatado observamos o envolvimento de T12 e L2.

O diagnóstico geralmente é realizado por meio da detecção de alterações típicas dos exames de imagem. Na radiografia simples a lesão na coluna pode apresentar- se como estrias verticais paralelas formadas pela dissociação das trabéculas ósseas sem alargamento do corpo vertebral, o que é considerado patognomônico. Pela TC é possível observar as traves ósseas esparsas alternadas com áreas hipertransparentes em cortes axiais. A RM demonstra sinais hiperintensos nos corpos vertebrais lesados, 
devido à substituição gordurosa ${ }^{(3)}$. Padrões radiológicos semelhantes são encontrados na osteoporose, doença de Paget e osteodistrofia renal.

Os hemangiomas, como todos os pequenos vasos e capilares, possuem moderada radiossensibilidade. Quando submetido à radiação, o endotélio dos pequenos vasos apresenta alterações precoces como degeneração, edema e necrose. As alterações radioinduzidas tardias dos pequenos vasos estão relacionadas à resposta do tecido conectivo intersticial, levando a fibrose e conseqüente estenose desses vasos ${ }^{(\mathbf{1 1})}$. Sabe-se que as alterações tardias, as quais são mais permanentes e efetivas, estão diretamente relacionadas com o aumento da dose por fração ${ }^{(\mathbf{1 2})}$

Vários métodos são descritos no tratamento do hemangioma vertebral. Nos casos de compressão medular, cirurgia descompressiva seguida ou não de radioterapia tem sido o tratamento de escolha ${ }^{(\mathbf{1 3 - 1 5})}$, a despeito de alguns autores preconizarem radioterapia exclusiva por causa dos riscos de sangramento intra-operatório ${ }^{(\mathbf{1}, \mathbf{1 6})}$. Yang et al. ${ }^{(\mathbf{1 7})}$ relataram que cinco de sete pacientes paraplégicos obtiveram remissão completa do quadro com radioterapia exclusiva na dose de 30 a 40 Gy durante quatro a seis semanas. Quadros associados a dorsalgia e sem déficits neurológicos podem ser conduzidos mediante observação periódica e tratamento clínico, sendo os casos refratários encaminhados à radioterapia exclusiva. Neste caso descrito, a paciente apresentava queixa de dorsalgia há um ano, a qual fora refratária ao tratamento com correção postural através do uso de colete ortopédico e antiinflamatório não-hormonal.

Em virtude do pequeno número de pacientes e séries publicados, não há discussão detalhada com relação à dose e regimes propostos. Manning ${ }^{(\mathbf{1 6})}$ preconizava que cursos repetidos de irradiação seriam necessários a fim de se obter um efeito adequado. Entretanto, Yang et al. ${ }^{(17)}$ notaram que somente um em seis pacientes obteve remissão completa da dor com múltiplos cursos de irradiação, com 10 a 20 Gy por curso. O efeito de cursos múltiplos com doses mais baixas não foi melhor do que o efeito de um único curso com dose mais alta, com cerca de 30 a 40 Gy.
Os esquemas mais utilizados na radioterapia do hemangioma são baseados em doses de 30 Gy em dez frações ou $40 \mathrm{~Gy}$ em 20 frações, sendo que $60 \%$ a $80 \%$ dos pacientes obtêm permanente e significante melhora dos sintomas como dor, paresia e paralisia ${ }^{(\mathbf{5 , 7}, 17)}$. Heyd et $_{\text {al. }}{ }^{(\mathbf{1 8})}$, em revisão da literatura, encontraram 59 relatos no período compreendido entre 1929 e 2000 , totalizando 327 casos. A análise de 55 trabalhos que relataram um total de 210 casos, nos quais se utilizou radioterapia exclusiva ou associada a outros métodos de tratamento, demonstrou que em 54\% dos casos houve remissão completa dos sintomas, em $32 \%$ obteve-se remissão parcial e em $11 \%$ não houve resposta.

$\mathrm{O}$ período de seguimento deste caso descrito foi de três meses, ao final do qual a paciente referia remissão parcial da dor. Não esperamos alteração do padrão radiológico após o tratamento. Apesar de serem indispensáveis para o diagnóstico do hemangioma vertebral, os exames de imagem não são úteis na avaliação dos efeitos da radioterapia. Sakata et al. ${ }^{(\mathbf{9})}$ relataram que nenhum dos pacientes submetidos à radioterapia demonstrou qualquer alteração da vértebra acometida através da radiografia simples, TC ou RM, mesmo após cinco anos de tratamento.

Faria et al. ${ }^{(\mathbf{5})}$ trataram nove pacientes ao longo de cinco anos, com dose de 30 a 40 Gy em frações diárias de $2 \mathrm{~Gy}$, cinco dias por semana, e $77 \%$ dos pacientes obtiveram melhora permanente ou remissão dos sintomas relacionados ao hemangioma vertebral num período de seguimento compreendido entre seis e 62 meses. Sugeriram ser a dose total de 40 Gy a mais adequada, pois, além de ser a dose máxima permitida, em virtude da dose de tolerância da medula espinhal, quanto maior a dose, maior o dano vascular.

No presente caso utilizamos três campos posteriores, com a finalidade de diminuirmos a dose total na medula através do campo posterior direto. Os dois campos oblíquos foram angulados em $60^{\circ}$, com o propósito de evitarmos a irradiação dos rins, haja vista que a dose de tolerância para um risco de $50 \%$ de complicações em cinco anos, quando dois terços do órgão são irradiados, é de $40 \mathrm{~Gy}$.
Concordamos que a dose total de $40 \mathrm{~Gy}$ em frações diárias de 2 Gy seja a mais adequada na radioterapia exclusiva do hemangioma vertebral, sendo que o quadro clínico é o único parâmetro disponível para a avaliação da resposta à radioterapia.

\section{REFERÊNCIAS}

1. McAllister VL, Kendall BE, Bull JW. Symptomatic vertebral haemangiomas. Brain 1975;98:7180.

2. Paige ML, Hemmati M. Spinal cord compression by vertebral hemangioma. Pediatr Radiol 1977;6: 43-5.

3. Murphey MD, Fairbairn KJ, Parman LM, Baxter KG, Parsa MB, Smith WS. From the archives of the AFIP. Musculoskeletal angiomatous lesions: radiologic-pathologic correlation. Radiographics 1995; 15:893-917.

4. Glanzmann C, Rust M, Horst W. Radiotherapie bei Angiomen der Wirbelsaule, Ergebnisse bei 62 Patienten aus dem Zeitraum 1939-1975. Strahlentherapy 1977;153:522-5.

5. Faria SL, Schlupp WR, Chiminazzo H Jr. Radiotherapy in the treatment of vertebral hemangiomas. Int J Radiat Oncol Biol Phys 1985;11:38790.

6. Eisenstein S, Spiro F, Browde S, Allen CM, Grobler $\mathrm{L}$. The treatment of a symptomatic vertebral hemangioma by radiotherapy. A case report. Spine 1986;11:640-2.

7. Guedea F, Majó J, Guardia E, Canals E, CravenBartle $\mathrm{J}$. The role of radiation therapy in vertebral hemangiomas without neurological signs. Int Orthop 1994;18:77-9.

8. Próspero JD. Neoplasias vasculares. In: Próspero JD, ed. Tumores ósseos. 1a ed. São Paulo, SP: Roca, 2001:175-95.

9. Sakata K, Hareyama M, Oouchi A, et al. Radiotherapy of vertebral hemangiomas. Acta Oncol 1997;36:719-24.

10. Karlin CA, Brower AC. Multiple primary hemangiomas of bone. AJR 1977;129:162-4.

11. Casarett GW, Rubin P. Radiation histopathology. In: Casarett GW, Rubin P, eds. Clinical radiation pathology. 1st ed. Philadelphia, PA: WB Saunders, 1968:38-61.

12. Fletcher GH. Biological aspects of radiotherapy. In: Fletcher GH, ed. Textbook of radiotherapy. 3rd ed. Philadelphia, PA: Lea \& Febiger, 1980: 103-80.

13. Healy M, Herz DA, Pearl L. Spinal hemangiomas. Neurosurgery 1983;13:689-91.

14. Baker ND, Klein MJ, Greenspan A, Neuwirth M Symptomatic vertebral hemangiomas: a report of four cases. Skeletal Radiol 1986;15:458-63.

15. Nguyen JP, Djindjian M, Gaston A, et al. Vertebral hemangiomas presenting with neurologic symptoms. Surg Neurol 1987;27:391-7.

16. Manning HJ. Symptomatic hemangioma of the spine. Radiology 1951;56:58-65.

17. Yang ZY, Zhang LJ, Chen ZX, Hu HY. Hemangioma of the vertebral column. A report on twenty-three patients with special reference to functional recovery after radiation therapy. Acta Radiol Oncol 1985;24:129-32.

18. Heyd R, Strassmann G, Filipowicz I, Borowsky $\mathrm{K}$, Martin T, Zamboglou N. Radiotherapy in vertebral hemangioma. Roentgenpraxis 2001;53: 208-20. 